\title{
Editorial: The Role of Flower Color in Angiosperm Evolution
}

\author{
Eduardo Narbona ${ }^{1 *}$, Montserrat Arista ${ }^{2}$, Justen B. Whittall ${ }^{3}$, \\ Maria Gabriela Gutierrez Camargo ${ }^{4}$ and Mani Shrestha ${ }^{5,6,7}$
}

\begin{abstract}
${ }^{1}$ Department of Molecular Biology and Biochemical Engineering, Pablo de Olavide University, Seville, Spain, ${ }^{2}$ Department of Plant Biology and Ecology, Faculty of Biology, University of Seville, Seville, Spain, ${ }^{3}$ Department of Biology, College of Arts and Sciences, Santa Clara University, Santa Clara, CA, United States, ${ }^{4}$ Laboratory of Ecology and Evolution of Plant-Animal Interactions, Institute of Biosciences, São Paulo State University, Botucatu, Brazil, ${ }^{5}$ Disturbance Ecology, Bayreuth Center for Ecology and Environmental Research, University of Bayreuth, Bayreuth, Germany, ${ }^{6}$ School of Media and Communication, Royal Melbourne Institute of Technology University, Melbourne, VIC, Australia, ${ }^{7}$ Faculty of Information Technology, Monash University, Melbourne, VIC, Australia
\end{abstract}

Keywords: flower color diversity, anthocyanins, flower color evolution, pollination, selective pressure

\section{Editorial on the Research Topic}

\section{The Role of Flower Color in Angiosperm Evolution}

\section{OPEN ACCESS}

Edited by: Akira Kanno,

Tohoku University, Japan

Reviewed by:

Takashi Nakatsuka,

Shizuoka University, Japan

*Correspondence:

Eduardo Narbona enarfer@upo.es

Specialty section: This article was submitted to Plant Development and EvoDevo, a section of the journal

Frontiers in Plant Science

Received: 06 July 2021 Accepted: 24 August 2021 Published: 17 September 2021

Citation:

Narbona E, Arista M, Whittall JB, Camargo MGG and Shrestha $M$ (2021) Editorial: The Role of Flower

Color in Angiosperm Evolution.

Front. Plant Sci. 12:736998.

doi: 10.3389/fpls.2021.736998
Although angiosperms exhibit a wide range of variability in floral traits such as shape and size, flower color is a hallmark of angiosperm diversity. Since before Darwin's time, flower color has long been appreciated for its role in pollinator attraction (Sprengel, 1793; Mendel, 1866; Darwin, 1895; Faegri and van der Pijl, 1966; Proctor and Yeo, 1973). However, over the past few decades, a growing body of evidence suggests that flower color can be molded by a diversity of selective pressures. The rapid accumulation of flower color studies has spurred several thorough reviews (Winkel-Shirley, 2001; Koes et al., 2005; Rausher, 2008; Sobel and Streisfeld, 2013; Narbona et al., 2018; Sapir et al., 2021), but here we present the largest collection of investigations specifically focused on the role of flower color in angiosperm evolution.

This Research Topic is composed of 28 studies on the role of flower color in angiosperm evolution. These contributions include species living on nearly all continents plucked from most major branches of the angiosperm tree of life (Figure 1). Investigations span traditional scales in biology, from gene expression and biochemical profiles to pollinator perception and community assembly. Evolutionarily, studies range from within species flower color polymorphisms to macroevolutionary patterns of flower color evolution within and among genera. Ecologically, investigations span a diversity of plant communities including neotropical savannas, temperate serpentine seeps, subtropical mountains, and tropical dry forests.

Fundamentally, flower color depends on the underlying pigments and recent studies have begun to decipher the genetic basis of this pigment production. The most prevalent and variable pigments in flowers are the anthocyanins, which originate from the flavonoid biosynthetic pathway (Tanaka et al., 2008). Branches of this pathway produce other flavonoid compounds (e.g., flavonols, flavones, isoflavones, proanthocyanins, and catechins) that protect plants against a variety of environmental stressors such as pathogens, herbivores, drought, extreme temperatures, ultraviolet (UV) radiation, etc. (Pollastri and Tattini, 2011; Falcone Ferreyra et al., 2012; Jiang et al., 2016). Thus, certain floral colors not only affect pollinator attraction (or avoidance), but may also determine resistance to biotic and abiotic stresses (Strauss and Whittall, 2006; Landi et al., 2015). 

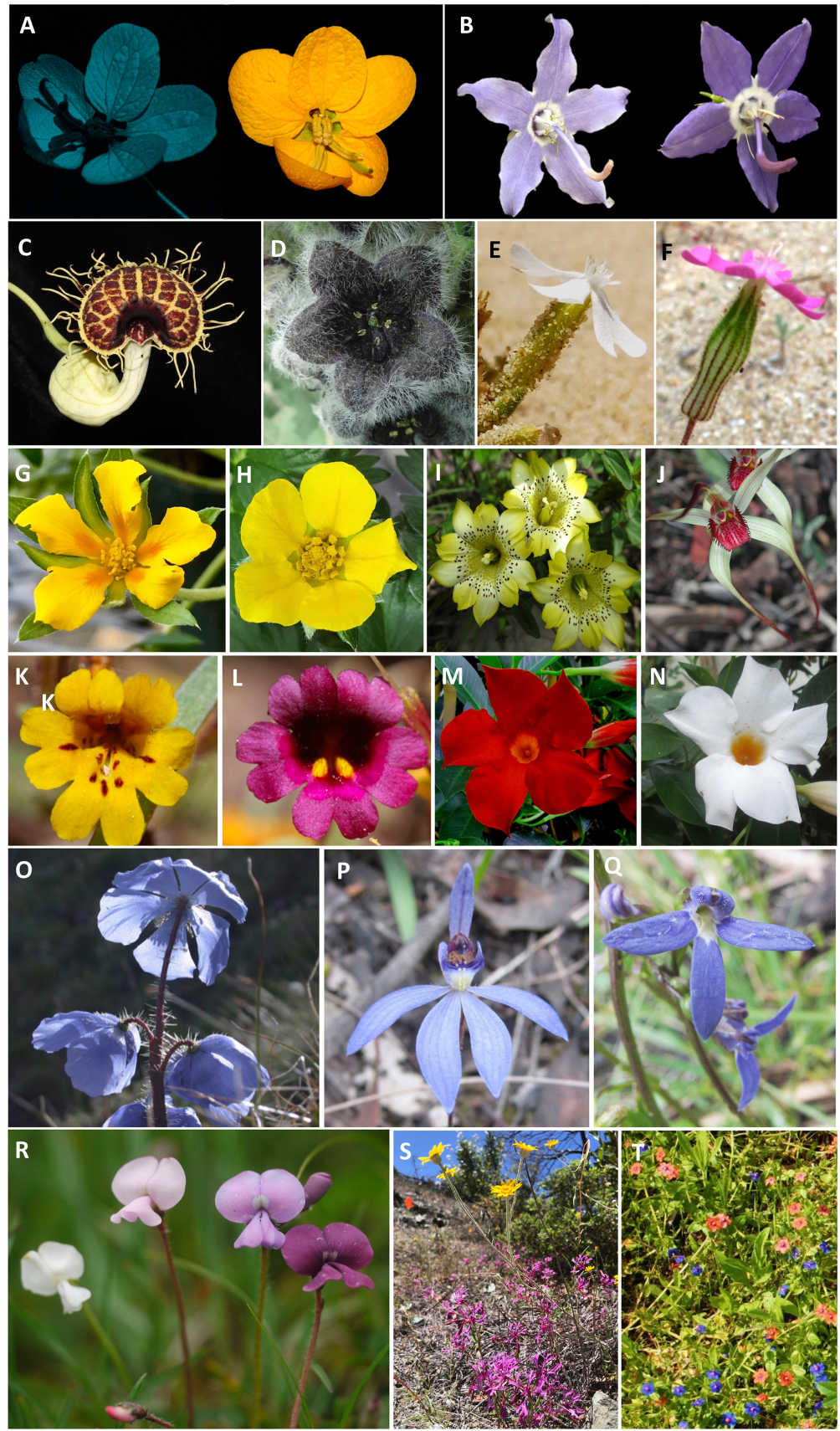

FIGURE 1 | Examples of species included in our Research Topic "The Role of Flower Color in Angiosperm Evolution." (A) Senna rugosa (Fabaceae) using UV (left) and conventional photography (right) (Brazil, Mariah di Stasi). (B) Flower color variation in Campanula americana (Campanulaceae, USA, Matthew Koski). (C) Aristolochia fimbriata (Aristolochiaceae, Colombia, Natalia Pabón-Mora). (D) Jaborosa rotacea (Gesneriaceae, Argentina, Marcela Moré). (E,F) White and pink flowers of Silene littorea plants (Caryophyllaceae, Spain, Eduardo Narbona). (G,H) Potentilla plattensis and Argentina anserine (Rosaceae, USA, Matthew Koski). (I) Gentiana flavomaculata (Gentianaceae, Taiwan, Chun-Neng Wang). (J) Caladenia fulva (Orchidadeae, Australia, Ann Lawrie). (K,L) Flower color morphs of Erythranthe discolor (Phrymaceae, USA, Naomi Fraga and Dena Grossenbacher). (M,N) Flower color morphs of Mandevilla sanderi (Apocynaceae, The Netherlands, Doekele Stavenga). (O) Meconopsis horridula (Papaveraceae, China, Anke Jenstsch). (P) Caladenia caerula (Orchidaceae, Australia, Mani Shrestha). (Q) Lobelia rhombifolia (Campanulaceae, Australia, Mani Shrestha). (R) Flower color variation in Tibetia yunnanensis (Leguminosae, China, Klaus Lunau). (S) Plants of Eriophyllum lanatum (Asteraceae) and Clarkia concinna (Onagraceae) coexisting (USA, Gerardo Arceo-Gómez). (T) Flower color morphs of Lysimachia arvensis coexisting (Primulaceae, Spain, Montserrat Arista). 


\section{THE GENETICS AND BIOCHEMISTRY OF ANTHOCYANIN PRODUCTION}

The anthocyanin biosynthetic pathway is mostly regulated at the transcriptional level (Albert et al., 2014). In this volume, Muñoz-Gómez et al. studied the regulatory gene evolution in the anthocyanin biosynthetic pathway among the Aristolochiaceae, a family with a high floral diversity exhibiting elaborate color patterns. The authors conclude that the anthocyanin biosynthetic pathway and its regulatory genes are largely conserved across the family, and color variation is primarily determined by differences in gene expression. Stavenga et al. studied the $\mathrm{pH}$ dependence of flavonoid absorption spectra of two Papaveraceae species, Papaver dubium (red) and Meconopsis cambrica (orange), and white and red varieties of Mandevilla sanderi. The authors found that the absorption spectrum (i.e. colour) of anthocyanins can be dependent on the $\mathrm{pH}$ of the vacuole, however, not all flavonoids respond similarly to changes in vacuolar $\mathrm{pH}$. Color diversity is also remarkably high in Gesnerioideae and Ogutchen et al. have elegantly shown that this is due to the expanded use of the anthocyanin biosynthetic pathway. In this family, the anthocyanin biosynthetic pathway includes the deoxyanthocyanin branch, which is rarely functional in angiosperms. Ogutchen et al. also call for a better understanding of the link between the biochemical basis of flower color and the visual perception of the primary pollinators. At the microevolutionary scale, Sánchez-Cabrera et al. identified several anthocyanin biosynthetic pathway loci likely involved in the shift in flower color in the orange/blue polymorphic Lysimachia arvensis. Further, these authors found differential expression of two genes $\left(F 3^{\prime} 5^{\prime} H\right.$ and $\left.D F R\right)$ in the anthocyanin biosynthetic pathway. The biochemical analysis of color morphs was consistent with the transcriptome data indicating that the shift from blue to orange petals is caused by a change from primarily malvidin to largely pelargonidin forms of anthocyanins (Sánchez-Cabrera et al.). Authors discuss that both the decreased expression of $F 3^{\prime} 5^{\prime} H$ in orange petals and the differential expression of two distinct copies of DFR, which also exhibit amino acid changes in the color-determining substrate specificity region, strongly correlate with the blue to orange transition. Collectively, these studies have revealed the complexity and nuance of the pigment biochemistry, yet the consistent role of gene expression changes in the anthocyanin biosynthetic pathway underlying the diversity of flower colors.

\section{HOW IS COLOR PERCEIVED?}

Flower color is in the eye of the beholder. Color perception depends on the sensory abilities of different groups of pollinators (van der Kooi et al., 2021). Flower color variation can arise when pollinators with diverse sensory systems drive fitness differences between flower color variants (Koski, 2020). Thus, in order to understand the selective role of pollinators in flower color, it is necessary to incorporate their color perception. Garcia et al. shows that experienced pollinators often make correct decisions about the presence of rewards based on flower color, but this is not the case for inexperienced pollinators. These authors concluded that color cannot be considered an inherent trait because its interpretation by an animal's brain is frequently context-dependent.

Although most studies of color perception and foraging decisions have been traditionally based on Apis mellifera's sensory system (e.g., Giurfa et al., 1994; Dyer et al., 2008; Rohde et al., 2013), we are in dire need of experimental studies on the sensory capabilities of non-Apis bee pollinators. In this volume, Koethe et al. have carried out a comparative study of food source selection between two stingless bee species and honeybees. These three species reacted similarly to color, but the variation among them could be the result of adaptations to the bees' respective habitat and morphological constraints. Thus, habitat traits can influence color perception by pollinators. In a similar conceptual framework, Martins et al. studied how seasonal changes in the leaf-background colouration of Brazilian savanna communities affect the perception of flower color contrasts by bees. They found that background coloration affected flower contrasts, favoring flower conspicuousness to bees according to the season and providing new insights regarding the temporal patterns of plantpollinator interactions.

Dyer et al. reviewed the old concept of the rarity of blue flowers. They found that short wavelength reflecting blue flowers are indeed frequent in nature when considering the color vision of bees and they point out that competition for pollinators may drive the evolution of blue flowers. Coimbra et al. used the visual system of bees to test the generalization of the bee-avoidance hypothesis proposed to explain why bird pollinated flowers tend to be red. Their results suggest that bee sensory exclusion via color signals is exclusive to bird flowers, while non-bee, insect flowers might use other sensory channels to exclude bees. In another study, Whitney et al. analyzed how flower color variation within plant populations of bee- and hummingbird-pollinated plant species is perceived by their specific pollinators. They found that bees sensed equal color variation within species from the two pollination systems, but birds perceived more color variation in bird-pollinated flowers than in bee-pollinated flowers.

\section{FLOWER COLOR VARIATION: A BROAD SCALE APPROACH}

Phylogenetically controlled studies at the community level have repeatedly found that flower color tends to show weak phylogenetic signal, reflecting an underlying pattern of evolutionary divergence (e.g., McEwen and Vamosi, 2010; Muchhala et al., 2014; Ortiz et al., 2021). For example, Tai et al. studied flower color signaling in Taiwan and found that although high altitude floras tend to be phylogenetically clustered, their flower colors exhibited only weak phylogenetic signal. Thus, they suggest that flower color signaling was mainly influenced by color preferences of key bee pollinators. Most studies on the phylogenetic signal of flower color focus on the predominant color exhibited by the flowers, although in many species flowers are not uniform showing contrasting colors (i.e., color patterns) that are perceived by pollinators (Hempel de Ibarra et al., 
2015). Some color patterns include reflection of UV light that is perceived by pollinators that have UV photoreceptors (van der Kooi et al., 2021). When floral color patterns in the ultraviolet spectrum (UV patterns) are investigated, contrasting results have been found. Tunes et al. studied patterns of floral UV reflectance in plants from a Neotropical savanna. They tested the roles of phylogenetic relatedness and pollinator mediated selection on the distribution of UV floral patterns. They confirmed that phylogenetic relatedness constrains the diversity of floral UV patterns, however, the distribution of floral UV-features could not be ascribed to a single ecological or evolutionary factor. This study calls for a deeper understanding of ecological and evolutionary processes involved when interpreting floral UV color diversification. Some flowers of the Potentilleae tribe (Rosaceae) have petals with UV patterns, whereas others show human-visible patterns or uniform petal color. Koski investigated the evolutionary transition between patterned and non-patterned petals and found that the presence of UV and human-visible patterns evolved independently from one another. He also found that the evolution of human-visible patterns was associated with the evolution of larger flowers, supporting the hypothesis that nectar or pollen guides are more likely to evolve in largerflowered species. In another study, Roguz et al. explored the evolution of flower color in Iris, a genus displaying a huge diversity of flower color and color patterns among and within species. They found that the most recent common ancestor likely had entomophilous, monochromatic flowers.

The fate of new color variants depends on a diversity of evolutionary forces. Flower color can affect pollinator attraction and, when investigated in a community context, flower color can mediate different types of interactions among co-flowering species, such as competition, facilitation or mimicry (Ghazoul, 2006; Johnson and Schiestl, 2016; Kemp et al., 2019). The abundance and species richness of the local habitat may also influence the type or strength of ecological interactions among species. Co-flowering species with similar flower colors may compete for pollinator services; selection could thus favor differentiation to improve pollinator recognition and fidelity (Gumbert et al., 1999; McEwen and Vamosi, 2010). Competition for pollinators would result in a pattern of phylogenetic overdispersion of floral color among co-flowering species (Sargent and Ackerly, 2008). Alternatively, similarity in flower color may result from selection for standardization of the signals that improves recognition by pollinators and increases their visitation rates. LeCroy et al. studied 14 serpentine seep communities in California differing in species richness and size. In smaller-sized communities they found that competitive exclusion could be a dominant process shaping lower species richness, but this process is less detectable in larger, more speciose communities. Similarly, Moré et al. explored the potential importance of pollinators as drivers of floral color diversification in the genus Jaborosa taking into account the perceptual abilities of their pollinators (i.e., nocturnal hawkmoths versus diurnal saprophilous flies) with a geographical perspective. This study found that the ability of plants that colonized the newly formed environments during Andean orogeny and the ecological changes that followed were concomitant with transitions in flower color as perceived by different pollinator functional groups. Further, this study suggests that habitat and pollination are linked to the history of a plant's lineage.

\section{FLOWER COLOR VARIATION AT THE MICROEVOLUTIONARY SCALE}

From a microevolutionary point of view, intraspecific variation in floral color is widely distributed (Narbona et al., 2018). Flower color may vary continuously or discretely, the latter situation being much rarer. Evolutionary mechanisms explaining the maintenance of intraspecific flower color variation include both biotic and abiotic factors, although other processes can also play an important role (Narbona et al., 2018). Trunschke et al. reviewed studies addressing selection on continuous flower color variation in the context of pollinator interactions. They suggest that evidence for significant pollinator-mediated selection is surprisingly limited among existing studies. In fact, most of the current understanding of flower color evolution arises from variation between discrete color morphs, where selection by pollinators is usually one of the most important factors involved in color variation. Selective foraging by pollinators for specific color morphs is frequently reported (Ortiz et al., 2015). These studies focus on the most common pollinators such as bees or birds, while selection by other groups is rarely reported.

Two studies in this volume have investigated microevolutionary flower color variation in South-African species pollinated by less-commonly-reported pollinator groups. Ellis et al. studied patterns of color distribution of discrete white and orange daisy species pollinated by bombyliids. They found that the dominant pollinator in orange communities has strong preferences for orange flowers while the dominant pollinator in white communities exhibited an innate preference for white flowers. These findings demonstrate that landscapelevel flower color turnover is likely shaped by a strong qualitative geographic mosaic of bee-fly pollinators with divergent color preferences. Similarly, Johnson et al. found that patterns of color variation in Drosera cistiflora, a species pollinated by beetles, are associated with different pollinator communities. Given that these beetle species discriminate among color forms (von Witt et al., 2020), the authors conclude that beetle pollinators are a significant factor in the evolution of D. cistiflora flower color. This is one of the first reports of flower color selection by beetles (see Streinzer et al., 2019).

Although direct selection on flower color by pollinators has been widely explored, indirect selection on correlated traits is rarely reported (e.g., Gómez, 2000). Other floral traits can be correlated with flower color and thus, to understand the role of pollinators as selective agents on flower color it is necessary to consider such associations. The number of flowers per plant, a component of floral display, is one of the most important traits affecting pollinator attraction, and its association with flower color has been studied in Silene littorea (Rodríguez-Castañeda et al.) and Medicago sativa (Brunet et al.). Both studies show significant correlations 
between flower color and floral display and demonstrate significant directional selection on floral display that indirectly selects for flower color. These examples of correlational selection can partly explain flower color evolution in $M$. sativa and the maintenance of the flower color polymorphism in S. littorea.

The synthesis of floral pigments can also have pleiotropic effects on defensive plant compounds and consequently herbivores can play a role in flower color variation (Strauss and Whittall, 2006). Specifically, anthocyanin pigments in floral tissues can influence herbivore preference and performance (Irwin et al., 2003; Frey, 2004). In this volume, Sobral et al. found evidence of transgenerational effects of herbivory on flower color variation in Raphanus sativus. Epigenetic modifications due to herbivory influences the proportion of plants with anthocyanins in the following generation showing a link between biotic ecological interactions across generations and plasticity in flower color; with some exceptions this phenomenon is virtually undescribed in natural plant populations.

Abiotic factors also play an important role in flower color variation (Strauss and Whittall, 2006). Water availability, temperature and solar radiation can select flowers with higher pigment concentrations either directly or indirectly, giving rise to geographical patterns of flower color variation (Dalrymple et al., 2020). The role of abiotic factors on flower color variation is more frequently reported and, in this volume, it has been addressed in two monkeyflowers-Erythranthe discolor and Diplacus mephyticus (Grossenbacher et al.), Campanula americana (Koski and Galloway), and Drosera cistifolia (Johnson et al.). Drought stress is one of the most important factors predicting flower color across geographic ranges (Warren and Mackenzie, 2001; Arista et al., 2013). Grossenbacher et al. found a higher frequency of anthocyanin producing morphs in populations with reduced precipitation for two monkeyflower species as a consequence of the protective role of anthocyanins. However, after accounting for phylogeny, there was no evidence that drought stress leads to a macroevolutionary pattern in flower color across monkeyflowers. In Campanula americana local temperature appears to shape the geographical pattern of flower color intensity, although the genetic population structure seems to be driven by historical effects, an important factor rarely considered (Koski and Galloway). Conversely, other rarely studied, potential abiotic selective agents (such as soil type) do not seem to be related to the geographic pattern of color variation in Drosera cistifolia (Johnson et al.). The role of abiotic factors on flower color variation has also been reported in this volume. Peach et al. studied the effect of UV radiation on flower color variation in Clarkia unguiculata and found that populations growing in areas of high UV radiation showed higher anthocyanin concentration, a pattern previously reported (Del Valle et al., 2015). However, contrary to expectations, UV-absorbing floral patterns did not have a direct "pollen protection" function, highlighting the need for research on a wider range of taxa to understand the role of anthocyanins in flower protection against UV radiation [see Kay et al. (2020)]. These studies addressing the role of abiotic factors affecting flower color variation highlight the protective role of pigments under some conditions of abiotic stress, but also reveal the multifaceted evolutionary and ecological complexity underlying natural flower color variation.

Other less known factors involved in the maintenance of intraspecific flower color variation are also reported in this volume. Jiménez-López et al. explored the role of selfing as a way to maintain the flower color polymorphism in Lysimachia arvensis. They found that in Mediterranean populations, both biotic and abiotic factors select against one of the color morphs, but that increased selfing in this morph preserves the color variation within populations. One consequence of this mating system by flower color interaction is a decrease in genetic variation that could have macroevolutionary consequences.

Flower color also shows intrafloral variation giving rise to complex patterns that can attract and guide bee pollinators. Aguiar et al. studied intrafloral color variation in Cattleya walkeriana, and found a centripetally increasing spectral purity within the flowers of this bee pollinated orchid species. This intrafloral variation was unrelated to the development of floral structures, suggesting an important role of pollinator selection in the modularization of flower color.

Lastly, the importance of taking flower color into account in conservation strategies is highlighted in this special topic. Orchidaceae is one of the most threatened plant families, as it has very large numbers and proportions of endangered species worldwide. Intraspecific flower color variability in the endangered orchid Caladonia fulva has been suggested to be the result of hybridization with $C$. reticulata. Genetic and breeding studies by Basist et al. clearly shows that C. fulva is a flower color polymorphic species and conservation of the color variants is essential to maintain genetic diversity.

In this Research Topic, we present a modern synthesis of flower color studies. Clearly, understanding the evolution of flower colors in angiosperms requires a diversity of perspectives across scales, both ecological and evolutionary, from the molecular, biochemical, physiological, anatomical, organismal, population, and community levels. These studies show that flower color in plant populations and plant communities are often the result of a combination of biotic and abiotic selective pressures and are highly context dependent. They clearly indicate the need to incorporate new approaches and innovative methodologies when studying flower color evolution.

\section{AUTHOR CONTRIBUTIONS}

All authors contributed to the article and approved the submitted version. 


\section{FUNDING}

Research by MA, EN, JW, and MC was supported by the European Regional Development Fund (ERDF) and grants from the Spanish government (PID2020-116222GB-I00) and the Andalusian Regional Ministry of Economy, Knowledge, Business and University (PY18-3651, US-1265280, and UPO1261687). MC received scholarships from CAPES Coordination of Superior Level Staff Improvement (grant \#88887.583309/202000 Finance Code 001) and FAPESP São Paulo Research Foundation (grant \#2015/10754-8). Research by MS was funded

\section{REFERENCES}

Albert, N. W., Davies, K. M., Lewis, D. H., Zhang, H., Montefiori, M., Brendolise, C., et al. (2014). A conserved network of transcriptional activators and repressors regulates anthocyanin pigmentation in eudicots. Plant Cell 26, 962-980. doi: 10.1105/tpc.113.122069

Arista, M., Talavera, M., Berjano, R., and Ortiz, P. L. (2013). Abiotic factors may explain the geographical distribution of flower colour morphs and the maintenance of colour polymorphism in the scarlet pimpernel. J. Ecol. 101, 1613-1622. doi: 10.1111/1365-2745.1 2151

Dalrymple, R. L., Kemp, D. J., Flores-Moreno, H., Laffan, S. W., White, T. E., Hemmings, F. A., et al. (2020). Macroecological patterns in flower colour are shaped by both biotic and abiotic factors. New Phytol. 228, 1972-1985. doi: $10.1111 /$ nph.16737

Darwin, C. (1895). The Effects of Cross and Self-Fertilisation in the Vegetable Kingdom. New York, NY: D. Appleton.

Del Valle, J. C., Buide, M. L., Casimiro-Soriguer, I., Whittall, J. B., and Narbona, E. (2015). On flavonoid accumulation in different plant parts: variation patterns among individuals and populations in the shore campion (Silene littorea). Front. Plant Sci. 6:939. doi: 10.3389/fpls.2015.00939

Dyer, A. G., Spaethe, J., and Prack, S. (2008). Comparative psychophysics of bumblebee and honeybee colour discrimination and object detection. J. Comp Physiol. A 194:617. doi: 10.1007/s00359-008-0335-1

Faegri, K., and van der Pijl, L. (1966). The principles of pollination ecology. Toronto Oxford 7, 157-159. doi: 10.1016/B978-0-08-023160-0.50020-7

Falcone Ferreyra, M. L., Rius, S. P., and Casati, P. (2012). Flavonoids: biosynthesis, biological functions, and biotechnological applications. Front. Plant Sci. 3:222. doi: $10.3389 /$ fpls.2012.00222

Frey, F. M. (2004). Opposing natural selection from herbivores and pathogens may maintain floral-color variation in Claytonia virginica (Portulacacea). Evolution 58, 2426-2437. doi: 10.1111/j.0014-3820.2004.tb00872.x

Ghazoul, J. (2006). Floral diversity and the facilitation of pollination. J. Ecol. 94, 295-304. doi: 10.1111/j.1365-2745.2006.01098.x

Giurfa, M., Núñez, J., and Backhaus, W. (1994). Odour and colour information in the foraging choice behaviour of the honeybee. J. Comp. Physiol. A 175, 773-779. doi: 10.1007/BF00191849

Gómez, J. M. (2000). Phenotypic selection and response to selection in Lobularia maritima: importance of direct and correlational components of natural selection. J. Evol. Biol. 13, 689-699. doi: 10.1046/j.1420-9101.2000.00196.x

Gumbert, A., Kunze, J., and Chittka, L. (1999). Floral colour diversity in plant communities, bee colour space and a null model. Proc. Royal Soc. London B 266, 1711-1716. doi: 10.1098/rspb.1999.0836

Hempel de Ibarra, N., Langridge, K. V., and Vorobyev, M. (2015). More than colour attraction: behavioural functions of flower patterns. Curr. Opin. Insect. Sci. 12, 64-70. doi: 10.1016/j.cois.2015.09.005

Irwin, R. E., Strauss, S. Y., Storz, S., Emerson, A., and Guibert, G. (2003). The role of herbivores in the maintenance of a flower color polymorphism in wild radish. Ecology 84, 1733-1743. doi: 10.1890/0012-9658(2003)0841733:TROHIT2.0.CO;2

Jiang, N., Doseff, A. I., and Grotewold, E. (2016). Flavones: from biosynthesis to health benefits. Plants 5:27. doi: 10.3390/plants5020027 by the German Federal Ministry of Education (BMBF) grant number 031B0516C and by Alan Dorin and Adrian G. Dyer under the funding scheme from the Australian Research Council Discovery Project 160100161.

\section{ACKNOWLEDGMENTS}

We express our sincere gratitude to all authors of papers published in this Research Topic for their enthusiasm and dedication. We thank a thoughtful reviewer and editor for helping to improve this Editorial.

Johnson, S. D., and Schiestl, F. P. (2016). Floral Mimicry. Oxford: Oxford University Press. doi: 10.1093/acprof:oso/9780198732693.001.0001

Kay, K. M., Jogesh, T., Tataru, D., and Akiba, S. (2020). Darwin's vexing contrivance: a new hypothesis for why some flowers have two kinds of anther. Proc. R. Soc. B 287:20202593. doi: 10.1098/rspb.2020.2593

Kemp, J. E., Bergh, N. G., Soares, M., and Ellis, A. G. (2019). Dominant pollinators drive non-random community assembly and shared flower colour patterns in daisy communities. Ann. Bot. 123, 277-288. doi: 10.1093/aob/mcy126

Koes, R., Verweij, W., and Quattrocchio, F. (2005). Flavonoids: a colorful model for the regulation and evolution of biochemical pathways. Trends Plant Sci. 10, 236-242. doi: 10.1016/j.tplants.2005.03.002

Koski, M. H. (2020). The role of sensory drive in floral evolution. New Phytol. 227, 1012-1024. doi: 10.1111/nph.16510

Landi, M., Tattini, M., and Gould, K. S. (2015). Multiple functional roles of anthocyanins in plant-environment interactions. Environ. Exp. Bot. 119, 4-17. doi: 10.1016/j.envexpbot.2015.05.012

McEwen, J. R., and Vamosi, J. C. (2010). Floral colour versus phylogeny in structuring subalpine flowering communities. Proc. Royal Soc. B 277, 2957-2965. doi: 10.1098/rspb.2010.0501

Mendel, G. (1866). Versuche über Pflanzen-hybriden. Verh. Natforsch. Ver. Brünn 4, 3-47. doi: 10.5962/bhl.title.61004

Muchhala, N., Johnsen, S., and Smith, S. D. (2014). Competition for hummingbird pollination shapes flower color variation in Andean Solanaceae. Evolution 68, 2275-2286. doi: 10.1111/evo.12441

Narbona, E., Wang, H., Ortiz, P. L., Arista, M., and Imbert, E. (2018). Flower colour polymorphism in the Mediterranean Basin: occurrence, maintenance and implications for speciation. Plant Biol. 20, 8-20. doi: 10.1111/plb.12575

Ortiz, P. L., Berjano, R., Talavera, M., Rodríguez-Zayas, L., and Arista, M. (2015). Flower colour polymorphism in Lysimachia arvensis: how is the red morph maintained in Mediterranean environments? Persp. Plant Ecol. Evol. Syst. 17, 142-150. doi: 10.1016/j.ppees.2015.01.004

Ortiz, P. L., Fernández-Díaz, P., Pareja, D., Escudero, M., and Arista, M. (2021). Do visual traits honestly signal floral rewards at community level? Funct. Ecol 35, 369-383. doi: 10.1111/1365-2435.13709

Pollastri, S., and Tattini, M. (2011). Flavonols: old compounds for old roles. Ann. Bot. 108, 1225-1233. doi: 10.1093/aob/mcr234

Proctor, M., and Yeo, Y. (1973). The Pollination of Flowers. Glasgow: William Collins.

Rausher, M. D. (2008). Evolutionary transitions in floral color. Intern. J. Plant Sci. 169, 7-21. doi: 10.1086/523358

Rohde, K., Papiorek, S., and Lunau, K. (2013). Bumblebees (Bombus terrestris) and honeybees (Apis mellifera) prefer similar colours of higher spectral purity over trained colours. J. Comp. Physiol. A 199, 197-210. doi: 10.1007/s00359-012-0783-5

Sapir, Y., Gallagher, M. K., and Senden, E. (2021). What maintains flower colour variation within populations?. Trends Ecol. Evol. 36, 507-519. doi: 10.1016/j.tree.2021.01.011

Sargent, R. D., and Ackerly, D. D. (2008). Plant-pollinator interactions and the assembly of plant communities. Trends Ecol. Evol. 23, 123-130. doi: 10.1016/j.tree.2007.11.003

Sobel, J., and Streisfeld, M. A. (2013). Flower color as a model system for studies of plant evo-devo. Front. Plant Sci. 4:321. doi: 10.3389/fpls.2013.00321 
Sprengel, C. K. (1793). "Das entdeckte Geheimniss der Natur im Bau und in der Befruchtung der Blumen. English translation by P. 1996," in Ecology and Evolution of Flowers, eds L. D. Harder and S. C. H. Barrett (Oxford: Oxford University Press), 3-43. doi: 10.5962/bhl.title.13716

Strauss, S. Y., and Whittall, J. B. (2006). "Non-pollinator agents of selection on floral traits," in Ecology and Evolution of Flowers, eds L. D. Harder, and S. C. H. Barrett (Oxford: Oxford University Press), 120-138.

Streinzer, M., Roth, N., Paulus, H. F., and Spaethe, J. (2019). Color preference and spatial distribution of glaphyrid beetles suggest a key role in the maintenance of the color polymorphism in the peacock anemone (Anemone pavonina, Ranunculaceae) in Northern Greece. J. Comp. Physiol. A 205, 735-743. doi: 10.1007/s00359-019-01360-2

Tanaka, Y., Sasaki, N., and Ohmiya, A. (2008). Biosynthesis of plant pigments: anthocyanins, betalains and carotenoids. Plant J. 54, 733-749. doi: 10.1111/j.1365-313X.2008.03447.x

van der Kooi, C. J., Stavenga, D. G., Arikawa, K., Belušič, G., and Kelber, A. (2021). Evolution of insect color vision: from spectral sensitivity to visual ecology. Ann. Rev. Entomol. 66, 435-461. doi: 10.1146/annurev-ento-061720-071 644

von Witt, C. G., Anderson, B., Durbach, I. N., and Johnson, S. D. (2020). Breeding systems of floral colour forms in the Drosera cistiflora species complex. Plant Biol. 22, 992-1001. doi: 10.1111/plb.13159

Warren, J., and Mackenzie, S. (2001). Why are all color combinations not equally represented as flower-color polymorphisms? New Phytol. 151, 237-241. doi: 10.1046/j.1469-8137.2001.00159.x

Winkel-Shirley, B. (2001). Flavonoid biosynthesis: a colourful model for genetics, biochemistry, cell biology, and biotechnology. Plant Physiol. 126, 485-493. doi: $10.1104 /$ pp.126.2.485

Conflict of Interest: The authors declare that the research was conducted in the absence of any commercial or financial relationships that could be construed as a potential conflict of interest.

Publisher's Note: All claims expressed in this article are solely those of the authors and do not necessarily represent those of their affiliated organizations, or those of the publisher, the editors and the reviewers. Any product that may be evaluated in this article, or claim that may be made by its manufacturer, is not guaranteed or endorsed by the publisher.

Copyright (c) 2021 Narbona, Arista, Whittall, Camargo and Shrestha. This is an open-access article distributed under the terms of the Creative Commons Attribution License (CC BY). The use, distribution or reproduction in other forums is permitted, provided the original author(s) and the copyright owner(s) are credited and that the original publication in this journal is cited, in accordance with accepted academic practice. No use, distribution or reproduction is permitted which does not comply with these terms. 